\title{
AN IN VIVO EVALUATION OF SURFACE POLISHING OF TAN INTERMEDULLARY NAILS FOR EASE OF REMOVAL
}

\author{
J.S. Hayes ${ }^{1,3, \#, \text { D.I. Vos }}{ }^{1,2, \#}$, J. Hahn ${ }^{1}$, S.G. Pearce ${ }^{1}$, R.G. Richards ${ }^{1,3, *}$ \\ ${ }^{1}$ AO Research Institute, Davos, AO Foundation, Davos, Switzerland \\ ${ }^{2}$ Amphia Hospital, Breda, The Netherlands \\ ${ }^{3}$ School of Biosciences, Cardiff University, Wales, Great Britain
}

\begin{abstract}
Fractures of the tibia and femoral diaphysis are commonly repaired by intra-medullary (IM) nailing. Currently IM nails are available in either electropolished stainless steel (SS) or in Titanium-Aluminium-Niobium (TAN). After healing, removal of the nails still is common but removal of TAN IM nails often has complications whereas SS IM nails of the same design are less often associated with problems. We believe the differences in removal are due to the ability of TAN to promote strong bone on-growth. We have previously shown in vivo that polishing cortical screws reduces removal torque and the percentage of bone-implant contact. Therefore, we postulate that bony on-growth onto IM nails can be reduced by means of surface polishing, for ease of removal. Here we aim to compare the pull-out forces for removal of standard TAN (TAN-S) compared to experimental paste polished TAN (TAN-PP) IM nails from a bilateral non-fracture sheep tibia model after 12 months implantation. Histological analysis was also performed to assess tissue on-growth to the nails. We show that polishing significantly reduces $(p=0.05)$ the extraction force required for TAN IM nail removal. This effect in part is attributable to the distinct tissue-material reaction produced. For TAN$\mathrm{S}$ nails direct bone contact was observed while for TANPP nails a fibrous tissue interface was noted. Since TAN is preferred over SS for IM nailing due to superior biocompatibility and mechanical properties, we believe these findings could be used to recommend changes to current surface technologies of intramedullary nails to reduce complications seen with nail removal especially in rapidly growing bone in children.
\end{abstract}

Keywords: Titanium-7\%Aluminium-6\%Niobium, Intramedullary Nails, Implant removal, Surface modification, in vivo.

*Address for correspondence:

R.G. Richards

AO Research Institute Davos

AO Foundation

Clavadelerstrasse 8

CH-7270 Davos Platz, Switzerland

Telephone Number: +41 (0)81 4142441

E-mail: geoff.richards@aofoundation.org

\# J.S. Hayes and D.I. Vos contributed equally to this paper

\section{Introduction}

Intramedullary (IM) nailing is a widely established and accepted method in the treatment for fractures of the femur, tibia and humerus. However, controversy surrounds the indications for its removal (Toms et al., 1996; Boerger et al., 1999; Gösling et al., 2004; Karladani et al., 2007; Morshed et al., 2007). Removal of an IM nail in symptomatic patients is primarily due to pain experienced by the patient or infection of the device and adjacent tissues (Husain et al., 1996; Luhmann et al., 2003; Flynn et al., 2004; Hui et al., 2007). Removal can also be due to nail intrusion into a joint, implant breakage or non union of the fracture site. A large number of extractions still occur in asymptomatic patients, especially paediatric patients, in order to prevent subsequent growth disturbances (Peterson, 2005; Simanovsky et al., 2006; Vierhout et al., 2006; Gogi et al., 2006; Mutimer et al., 2007) and also as a prophylactic measure to prevent future complications in case of joint replacement (knee, hip or shoulder).

In the United Kingdom alone approximately $13 \%$ of all active members of the British Orthopaedic Association (BOA) routinely carry out IM nail removal surgery after uncomplicated healing (Zenios et al., 2004). Interestingly, however, is that a patient being asymptomatic was listed in the same study as a contraindication by most surgeons for nail removal (Zenios et al., 2004). Thus one can postulate that advocates of nail retention arise not because they wholly believe there are benefits for retaining the device rather than that the risks and costs associated with removal outweigh that surrounding nail retention. However, IM nail extraction in children has clearer indications. Within this subset of patients, nail removal is often warranted to avoid growth complications such as limb shortening and related growth disturbances (Peterson, 2005; Simanovsky et al., 2006; Vierhout et al., 2006; Gogi et al., 2006; Mutimer et al., 2007). Indeed, approximately $53 \%$ of BOA surgeons claim to routinely remove IM nails in asymptomatic paediatric patients (Zenios et al., 2004). In a more recent survey of 60 paediatric surgeons, 98\% claim to routinely remove devices (AO Trauma Course-Pediatric Fractures December 9-11, 2008, Davos, Switzerland).

Despite the low recorded number of complications related for this procedure one issue that has arisen is the excessive bony on-growth on nails that cause intraoperative complications. In a retrospective study of five cases of IM nail removal, complications associated with excessive bone on-growth were reported for all patients (Seligson et al., 1997). In only two of these cases was 
nail removal achieved upon primary attempt, however, both cases resulted in subsequent minimal fractures of the surrounding site due to difficult removals. For the remaining three cases removal was incomplete upon the first extraction attempt. All three cases resulted in the nail being re-inserted and only one of these cases subsequently resulted in a second successful removal attempt (Seligson et al., 1997). Therefore, difficulties associated with excessive bone on-growth are not only surgically related complications such as increased operative time, blood loss, patient trauma and possible re-fracture but can also produce device-related complications such as incomplete/failed removal, debris contamination, and implant/instrument failure (Husain et al., 1996; Bombaci and Gorgec, 2003). Whether it is the choice of the surgeon or the patient, fundamentally once fracture healing has occurred the function of an IM nail is spent. Therefore, no definitive scientific or clinical reason exists for retention of an IM nail. However, removal of titanium- $6 \%$ aluminium$7 \%$ niobium (TAN) IM nails often has complications whereas electropolished stainless steel (SS) IM nails of the same design are less often associated with removal problems (Im and Lee, 2003; Woodruff et al., 2003; Gösling et al., 2005). Since nail design and geometry are similar between these two nail types, we believe many of the problems to be surface related.

Previous in vitro work by our laboratory (Baxter et al., 2002; Meredith et al., 2007a; Meredith et al. 2007b; Hayes et al., 2007) and others (Boyan et al., 2001; Schneider et al., 2003) have highlighted the importance of surface microtopography in determining cell phenotype. Specifically we have shown that the surface of an implant can directly influence fibroblastic (soft tissue) and osteoblastic (hard tissue) behaviour (Baxter et al., 2002; Biggs et al., 2007; Meredith et al., 2007a; Meredith et al. 2007b; Hayes et al., 2007). In terms of hard tissue response we have shown that smoother surfaces compared to the clinically available 'standard' micro-rough counterparts hold potential for reducing bony over-growth by essentially reducing osteoblast 'specific' genotypic expression via alterations in the cell shape and cytoskeletal organization (Hayes et al., 2008). We have also observed this effect translated to a tissue level in vivo, where the importance of the surface characteristics of clinically used devices were found to directly control specific tissue outcomes (Welton, 2006; Pearce et al., 2008; Schlegel et al., 2009).

With these points in mind we believe that the removal difficulty reported for TAN IM nails is due to the excellent ability of this material to promote strong bone on-growth. Indeed the difficulties encountered during nail removal due to excessive bony on-growth have been reported (Seligson et al., 1997) but few feasible solutions have been put forth and experimental data remains restricted to animals (Miettinen et al., 1992) and human cadaveric samples (Roure et al., 1999). Nevertheless, TAN remains the preferred choice over SS IM nails partly due to issues related to SS pertaining to toxic metal ion release (Case et al., 1994; Hanawa, 2004) with the principal constituents of SS, chromium, cobalt and nickel, being the main culprits, mechanical inferiority under cyclic loading (TAN provides lower stiffness with higher fatigue strength compared to SS) (Perren et al., 2001; Eschbach et al., 2001), imaging disadvantages such as increased black spot artefacts for magnetic resonance imaging (Pohler, 2000; Eschbach, 2003) and second-rate biocompatibility (Massone et al., 1991; Nichols \& Puleo, 1997). Therefore, in an attempt to address this issue we hypothesise that a smooth surface created by polishing of standard micro-rough TAN nails will help to minimise the nail removal problems associated with excessive bone-on-growth and will require lower pull out forces than the standard nails with rough TAN surfaces. To this end, this study is aimed primarily at assessing the effect of polishing on the pull out forces required for the removal of standard TAN and paste polished TAN IM nails in a bilateral non-fracture sheep tibia model after a 12 month implantation. Prior to this evaluation, an in vivo proof of concept study was carried under similar conditions to test the pull-out force and histological response of SS versus standard TAN IM nails.

\section{Materials and Methods}

\section{Surface Characterisation}

Commercially available Synthes ${ }^{\circledR} 9.5 \mathrm{~mm}$ Universal Humeral Nails (UHN) made of shot-peened electropolished stainless steel (SS) (ISO 5832/1) or titanium-6\%aluminium-7\%niobium (TAN) (ISO 5832/11) with either a standard micro-rough surface (TAN-S) or an experimental paste-polished surface (TAN-PP) were used. Paste polishing is a mechanically abrasive technique used to reduce the surface microtopography of a material.

Samples were characterised with profilometry, scanning electron microscopy (SEM), contact angle and X-ray photoelectron spectroscopy (XPS; Meredith et al., 2007a; Meredith et al., 2007b). The samples were ultrasonically cleaned with ethanol for $10 \mathrm{~min}$ before being air-dried for analysis. Implant surface topography characterisation of each nail was assessed quantitatively using a non-contact white light FRT MicroProf ${ }^{\circledR}$ (Fries Research \& Technology, Bergisch Gladbach, Germany) on 3 test nails which were not implanted. A roughness average ( $\mathrm{Ra}$ - arithmetic mean of the roughness height expressed in micrometers) was measured from a $1 \times 1 \mathrm{~mm}$ scan area with a point density of 1000 points/line. The morphology of the nails surfaces were examined using a Hitachi S4100 field emission SEM (Hitachi HighTechnologies, Krefeld, Germany). The images were taken in backscattered electron mode with an accelerating voltage of $5 \mathrm{kV}$. Surface chemical analysis was carried out with XPS. All spectra were recorded on a Kratos Axis Nova (Kratos Analytical, Manchester, UK) using monochromated Al K $\alpha$ radiation (1486.69 eV). For quantification survey scans with a step width of $0.5 \mathrm{eV}$ were performed on two spots of $300 \times 700 \mu \mathrm{m}^{2}$ per sample. Data were evaluated with CasaXPS 2.3.10 (CasaXPS Ltd, UK; www.casaxps.com) using relative sensitivity factors supplied by the manufacturer. Hydrophobicity was evaluated with static contact angle measurements (Andrade, 1985) using the Sessil drop method with the 
Drop Shape Analysis System (Contact Angle Measuring Instrument G10 and DSA 10 Control Unit, Krüss GmbH, Hamburg, Germany) and analysed using the Drop Shape Analysis 1.50 software (Krüss). Samples were placed in a preheated chamber $\left(20^{\circ} \mathrm{C}\right)$ with an external water source to sustain a constant relative humidity. Using the computer controlled system a $20 \mu \mathrm{l}$ droplet of distilled water was dispensed onto the sample and quantified exactly 1 minute after dispensed using the analysis software.

\section{In Vivo Methods}

Approval to perform this study was granted by the Cantonal animal ethics committee (GR 5/2006). Fourteen adult female Swiss Alpine sheep were selected from a group of Swiss Alpine sheep subsequent to radiological analysis being preformed on both tibiae to control length and diameter of the tibia. Using a bilateral non-fracture model seven sheep were used to provide proof of concept by comparing SS versus TAN-S IM nails implanted within contralateral tibiae. To assess the influence of polishing on IM nail pull-out force and subsequent material-tissue interaction another seven sheep were then implanted with an S-TAN nail in one tibia and a PP-TAN nail in the contralateral tibia. During the course of the surgeries, within our proof of concept group (SS versus TAN-S), nails were locked proximally and distally in orthogonal planes using a $5.0 \mathrm{~mm}$ locking screw made from the same material and surface treatment as the nail. Locking was facilitated using a custom made aiming device attached by means of the threads in the proximal aspect of the nail that were used for attaching the insertion device and the endcap. However, observations in this initial study indicated that due to the close fit of the human nail in several anatomical areas of the reamed sheep intramedullary canal two interlocking screws were considered to be redundant to prevent migration of the nail and to avoid fracture of the highly reamed bone. Therefore, in the interest of reducing surgical time for the sheep and to minimise the chance of fracture the distal locking screw was not used in the experiment containing the S-TAN versus PP-TAN groups.

Animals were anaesthetised (diazepam (Valium $\left.{ }^{\circledR}\right) 0.3$ $\mathrm{mg} / \mathrm{kg}$ i.v. and butorphanol (Morphasol ${ }^{\circledR}$ ) $0.08 \mathrm{mg} / \mathrm{kg}$ i.v.; thiopenthal (Penthotal ${ }^{\circledR}$ ) 4-8 mg/kg i.v.; isoflurane (approx $2 \%$ in oxygen: oxygen flow rate was approx $0.5 \mathrm{~L} / \mathrm{min}$ )), and placed in the appropriate lateral recumbency such that the operated limb was dependent (the animal was rotated into the opposite recumbency for operation of the second limb). Both tibiae were prepared for aseptic surgery. Surgery for both groups of sheep involved a $5 \mathrm{~cm}$ incision being made on the medial aspect of the patellar ligament of the stifle joint for both hind limbs. Subsequently, the joint was flexed approximately 90 degrees in order to gain access to the proximal tibia. Approximately half way between the patellar tendon and the cranial edge of the medial femoral meniscus a hole was drilled into the joint surface of the proximal tibia. The tibia was reamed in increments of $0.5 \mathrm{~mm}$ to a maximum diameter of $11 \mathrm{~mm}$ with a rigid reamer in order to create a more uniform amount of contact between the nail and tibial cortex. The

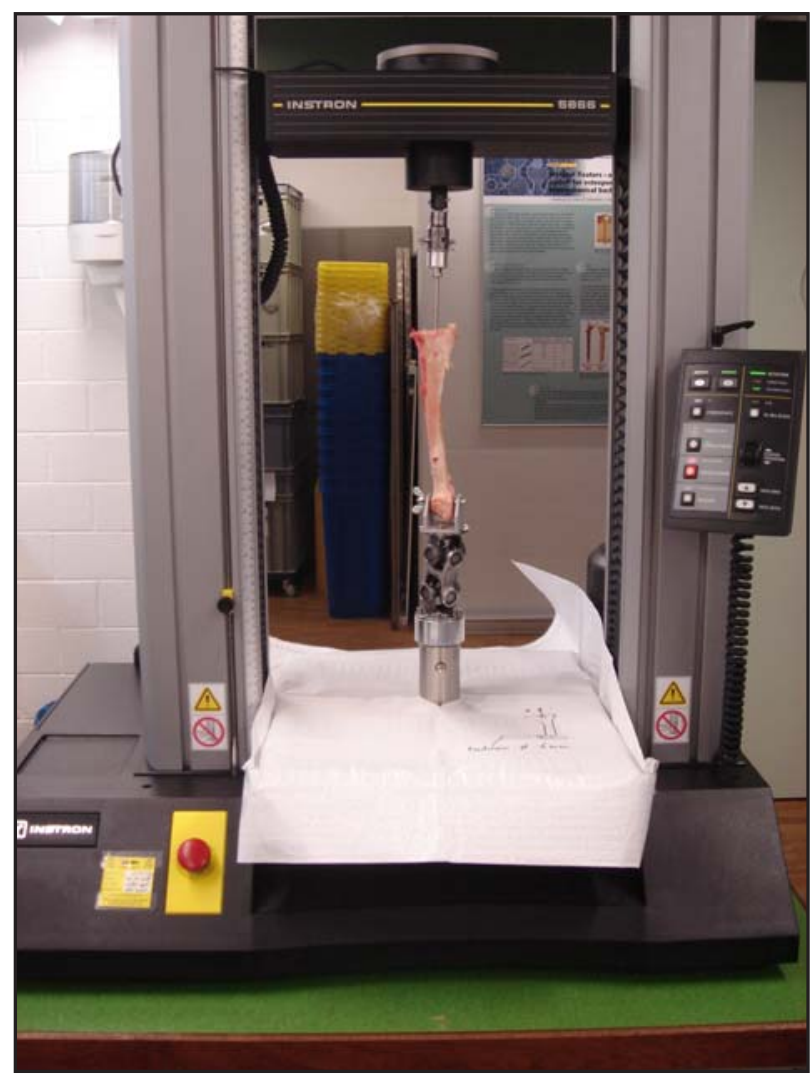

Figure 1. Testing Device, with adapter screwed into the endcap holder of the nail within the tibia before pull out with was performed with a constant force

tibia plateau was reamed to $12 \mathrm{~mm}$ to ease nail insertion. A human $9.5 \mathrm{~mm}$ diameter UHN (humeral nail) was inserted (with hammering) into the tibia through an extraarticular approach and a proximal locking bolt was inserted via a small incision through the skin and finally an endcap was placed at the proximal site of the nail.

The subcutaneous tissue was closed with absorbable suture material and the skin was closed with stainless steel skin staples. The limbs were bandaged with cotton wool and an adhesive bandage which was left in place for 5-7 days or less if believed to be causing irritation to the animal. Post-operatively analgesia consisted of carprofen $(4 \mathrm{mg}$ / $\mathrm{kg}$ subcutaneous, q24h for 3 days, Rimadyl ${ }^{\circledR}$, Pfizer, New York, NY, USA) and buprenorphine $(0.01 \mathrm{mg} / \mathrm{kg}$ subcutaneous, $\mathrm{q} 12 \mathrm{~h}$ for 3 days, Temgesic ${ }^{\circledR}$, ESSEX Chemie AG, Luzern, Switzerland). Immediately postsurgery and for the first week of the observation period all animals were housed in individual boxes in protection slings that allowed full weight bearing on the legs, but not reclining. No complications were encountered during or subsequent to surgery. Each sheep was evaluated every 4 weeks clinically for the duration of the study and every 8 weeks radiographs were taken in two plains latero-medial and cranio-caudal to monitor the nail position stability and any visible signs of bone on-growth. The nails were left in place for a total implantation time of 12 months. The animals were euthanized by means of an intravenous overdose of barbiturate (Pentobarbital, Vetanarcol ${ }^{\circledR} 60 \mathrm{mg} /$ $\mathrm{kg})$. 


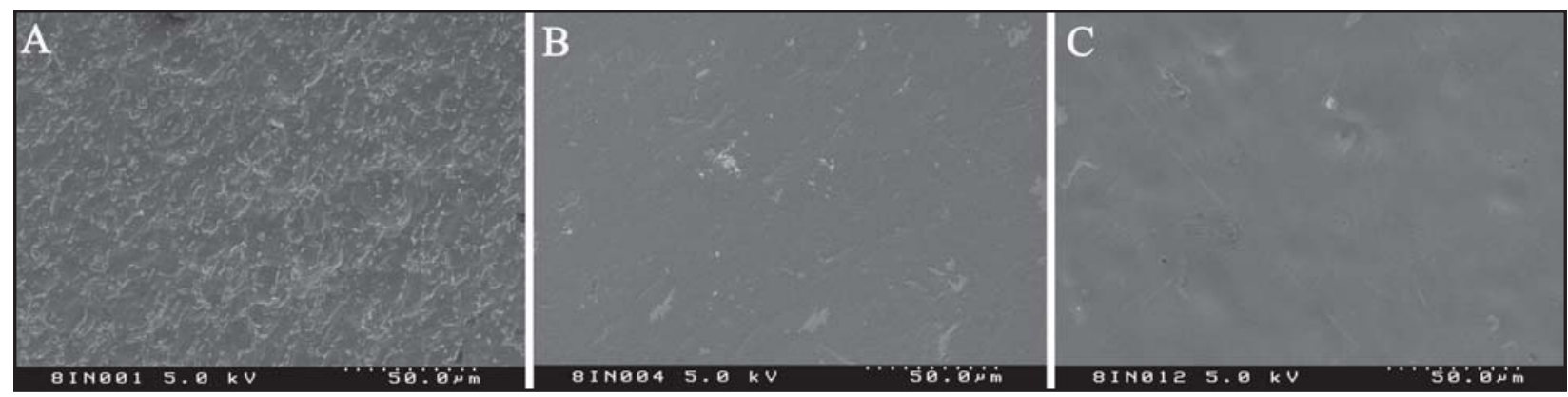

Figure 2. SEM images of (A) S-TAN showing a rough undulating surface with numerous micro-discontinuities (as used in clinics), (B) PP-TAN showing a smoothened surface with very few micro-discontinuities, (C) SS with a smooth, yet undulating surface (standard shot-peened surface, as used in clinics).

\section{Mechanical Testing}

After euthanasia 6 sheep from each the proof of concept study and also from the subsequent main study were used for the pull-out tests. The tibiae were excised, adjacent soft tissues removed and the tibia plateau was cut just to above the proximal position of the nail. Distally a $6 \mathrm{~mm}$ drill hole was made $2 \mathrm{~cm}$ from the distal central end and the endcap was removed to allow attachment to the testing device (Fig. 1). The locking bolts were removed from the nails after fixing the tibia in the pull-out machine. Subsequent pull-out tests were performed on these nails using a mechanical testing machine Instron 5866 (Instron Inc, High Wycombe, UK) with a $10 \mathrm{kN}$ load-cell and a custom made pull-out device. All nails were removed with a constant force $(1 \mathrm{~mm} / \mathrm{min})$. Load- and displacement-data was recorded with a sampling rate of $50 \mathrm{~Hz}$. Data were evaluated using Matlab ${ }^{\circledR}$ software (V6.5, Mathworks Inc, Natick, MA, USA) with a custom made procedure. In one sheep from each group the nail remained in situ without mechanical tests and was used for histomorphometric analyses.

\section{Histological Observations}

After euthanasia one nail from each group was left in situ. The whole intact tibia containing the nail was fixed in $70 \%$ methanol for approximately 9 months. Subsequently dehydration of the samples was achieved by immersing the samples in increasing concentrations of ethanol $(70 \%$, $96 \%$, and $100 \%$ ). Samples were then placed in xylol prior to being embedded with methylmethacrylate. Sections were cut with an annular saw, ground (Exact Micro Grinding System,Little Rock, AR, USA) and polished to a thickness of approximately $100 \mu \mathrm{m}$ and stained with Giemsa and eosin. Light microscopic images were taken using a Zeiss Axioplan microscope (Carl Zeiss, Göttingen, Germany).

\section{Statistical Analysis}

Statistical evaluation was performed using SPSS for Windows Version 14.0 (SPSS Inc, Chicago, Illinois, USA). Initially to provide proof of concept the SS versus TAN-S group were evaluated statistically using Wilcoxon signed ranks test given that the data for this group had a nonGaussian distribution. Subsequently, within our main group the effect of polishing with a paired model (TAN-S versus

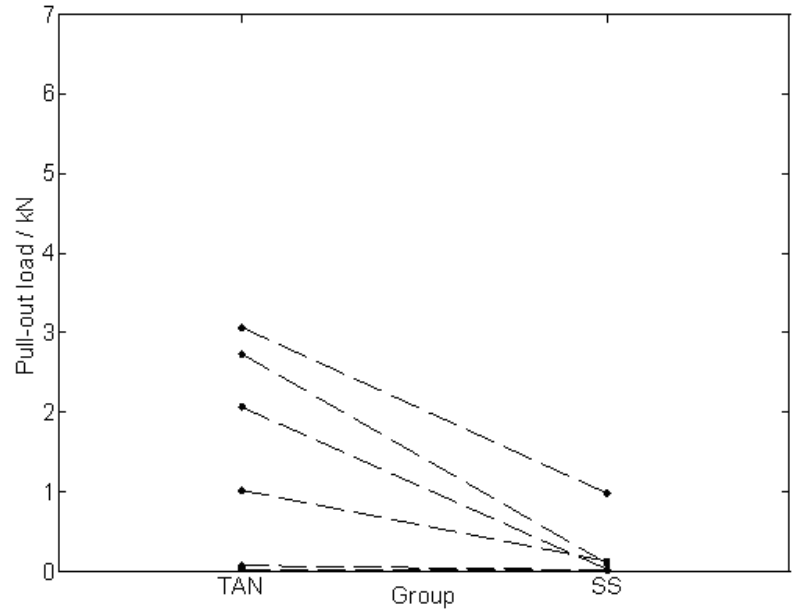

Figure 3. Pullout test results for the proof of concept study comparing S-TAN and SS nails in a paired test within individual sheep. As expected, SS IM nails were significantly easier to remove compared to S-TAN IM nails $(p=0.028)$. For two sheep in this group, the pullout force was very low in both tibiae. While no statistical correlation was found between intramedullary canal width, and pull-out force (S-TAN $p=0.345$; SS $p=0.133$ ), it was observed radiologically that the two specimens in question had a wider medullary canal than the other specimens, which will clearly influence the force required for nail removal.

TAN-PP) was assessed using a paired $t$-test, $p$-values $\leq 0.05$ were deemed significant.

\section{Results}

\section{Surface characterisation}

Non-contact profilometry revealed that the nails made of standard micro-rough TAN (S-TAN), shot-peened electropolished stainless steel (SS) and paste-polished TAN (PP-TAN) used in this study had an average surface roughness of $0.98 \mu \mathrm{m}, 0.58 \mu \mathrm{m}$ and $0.18 \mu \mathrm{m}$, respectively. The XPS results delineating the atomic concentration (\%) of elements of the nail surface were for S-TAN: $\mathrm{Al} 2.1 ; \mathrm{C}$ $26 ; \mathrm{N} 0.9 ; \mathrm{Na} 0.5 ; \mathrm{Nb} 0.2 ; \mathrm{O} 50.9$; P 2; Ti 17.2 and for PP- 


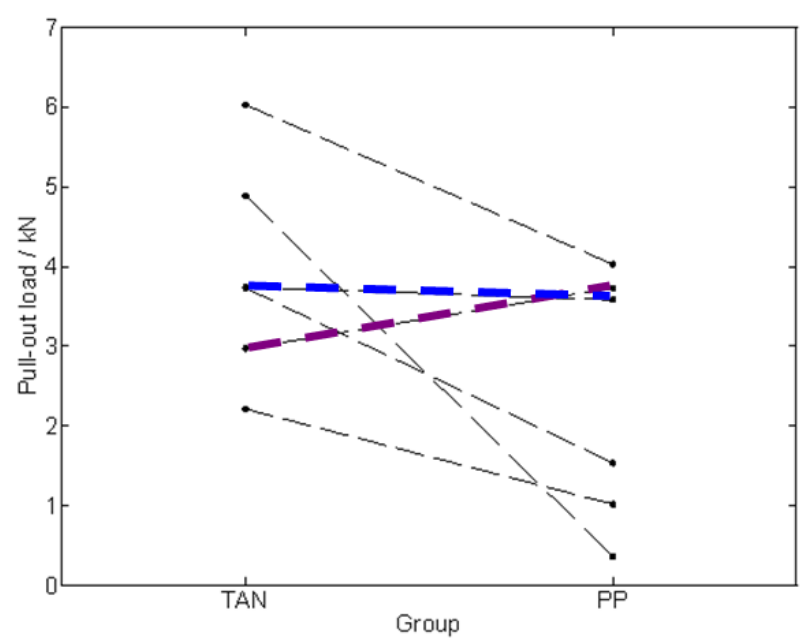

Figure 4. Pullout test results of the main study group comparing S-TAN and PP-TAN nails in a paired test within individual sheep. Results indicate that reducing the surface microtopography of TAN IM nails with paste polishing can significantly ease removal compared to S-TAN IM nails $(p=0.05)$. Two exceptions were noted. Firstly, a higher pullout force was noted for one PPTAN, (denoted with purple dashed line) compared to the contra-lateral tibia containing the S-TAN IM nail which was due to the tight fit of the nail to the medullary canal. Consequently the bone broke upon explantation. While no statistical correlation was observed between medullary canal width and pull-out force for PP-TAN IM nails $(p=0.323)$, radiologically the differences in medulla width was evident. Secondly, results from a different sheep produced similar pullout forces for both nails (denoted by blue dashed line). Radiological analysis showed bony in-growth of the lower hole of both the PP-TAN and S-TAN nails, so that a cylinder of bone of equal volume had to be broken for both nails, accounting for the similar pull forces noted. The data from this sheep were excluded from the statistical analysis.

TAN: Al 2.4; C 27.6; N 1.1; Na 0.6; Nb 0.1; O 49; P 2.5; Ti 16.5. Therefore polishing of the TAN nails followed by anodisation (PP-TAN) did not alter the chemical properties of the surface compared to S-TAN controls which were also anodised. Contact angle measurements gave an indication of the wettability (hydrophobic or hydrophilic) of a surface which also affects cell and tissue adhesion. Results were $78.3 \pm 6.6$ for SS, $64.2 \pm 7.7$ for S-TAN and $64.1 \pm 2.9$ for PP-TAN. SEM imaging clearly showed the characteristic $\beta$-phase niobium-rich inclusions associated with the micro-spiked appearance of the S-TAN (Fig. 2A). Paste polishing (Fig. 2B) clearly reduced the microtopography of the surface successfully to the extent that the niobium rich inclusions were not easily observed. Imaging of stainless steel (Fig. 2C) highlighted the importance of using various techniques for characterising surfaces. While non contact profilometry assesses the surface as being relatively rough ( $\mathrm{Ra} 0.58 \mu \mathrm{m})$, SEM imaging demonstrated that the actual surface was smooth with an undulating morphology; however, the waviness encountered on areas of the surface would contribute to the higher average roughness observed.

\section{Mechanical Testing}

To provide proof of concept mechanical pull-out tests of the SS nails and S-TAN nail from the same sheep were performed. Results demonstrated that as expected the SS nails had a significantly lower $(p=0.028)$ pullout force than the S-TAN nails (Fig. 3). For two sheep in this group the pullout force was very low in both tibiae. While no statistical correlation was found between intramedullary canal width and pull-out force (S-TAN $p=0.345$; SS $p=0.133$ ) it was observed radiologically that the two specimens in question had a much wider medullary canal than the other specimens which will clearly influence the force required for nail removal.

In our main group (TAN-PP versus TAN-S) designed at testing the influence of surface polishing of TAN IM nails mechanical pullout tests of the experimental PP-TAN nails also demonstrated a markedly lower pullout force compared to the S-TAN nail implanted in the same $(p=0.05)$ (Fig. 4). However within this group two exceptions were noted. Firstly, a higher pullout force was noted for one PP-TAN (Fig. 4) which was revealed to be slightly higher than the pull out force required for the contra-lateral tibia containing the S-TAN IM nail. Radiologically, it was noted that there was a cortical reaction for both tibiae containing PP-TAN and S-TAN nails. Additionally, during testing of the PP-TAN IM nail due to the tight fit of the nail to the medullary canal of this small sheep (over 200 sheep were examined radiologically however some were clearly smaller) the bone broke (Fig. 5A,B). Again no statistical correlation was observed between medullary canal width and pull-out force for PPTAN IM nails $(p=0.323)$; however, radiologically the differences in medulla width were evident. Secondly, pull out forces of PP-TAN and S-TAN from a different sheep produced similar results for both nails. Radiological analysis as well as analysis by eye after the pull out tests showed bony in-growth of the lower distal hole of both the PP-TAN and S-TAN nails (Fig. 5C) so that a cylinder of bone of equal volume had to be broken for both nails accounting for the similar pull forces noted. Therefore the results for the pullout test were a reflection of the force to break a cylinder of the bone in the hole rather than on the force required to remove the nail. The data from this sheep were excluded from the statistical analysis.

Bone was not observed to grow directly onto the surfaces of the nail interlocking holes of the polished nails and any tissue within the screw holes was easily displaced with a $\mathrm{K}$ wire (Fig. 6A). In contrast, within the interlocking holes of standard TAN nails bone on-growth onto the surface of the holes was evident and this hard tissue could not be displaced (Fig. 6B). Bone was also observed on the cutting flutes at the tip of the standard TAN locking bolts whereas for paste polished TAN locking bolts and stainless steel ones of the same design bone tissue adhesion was not observed 

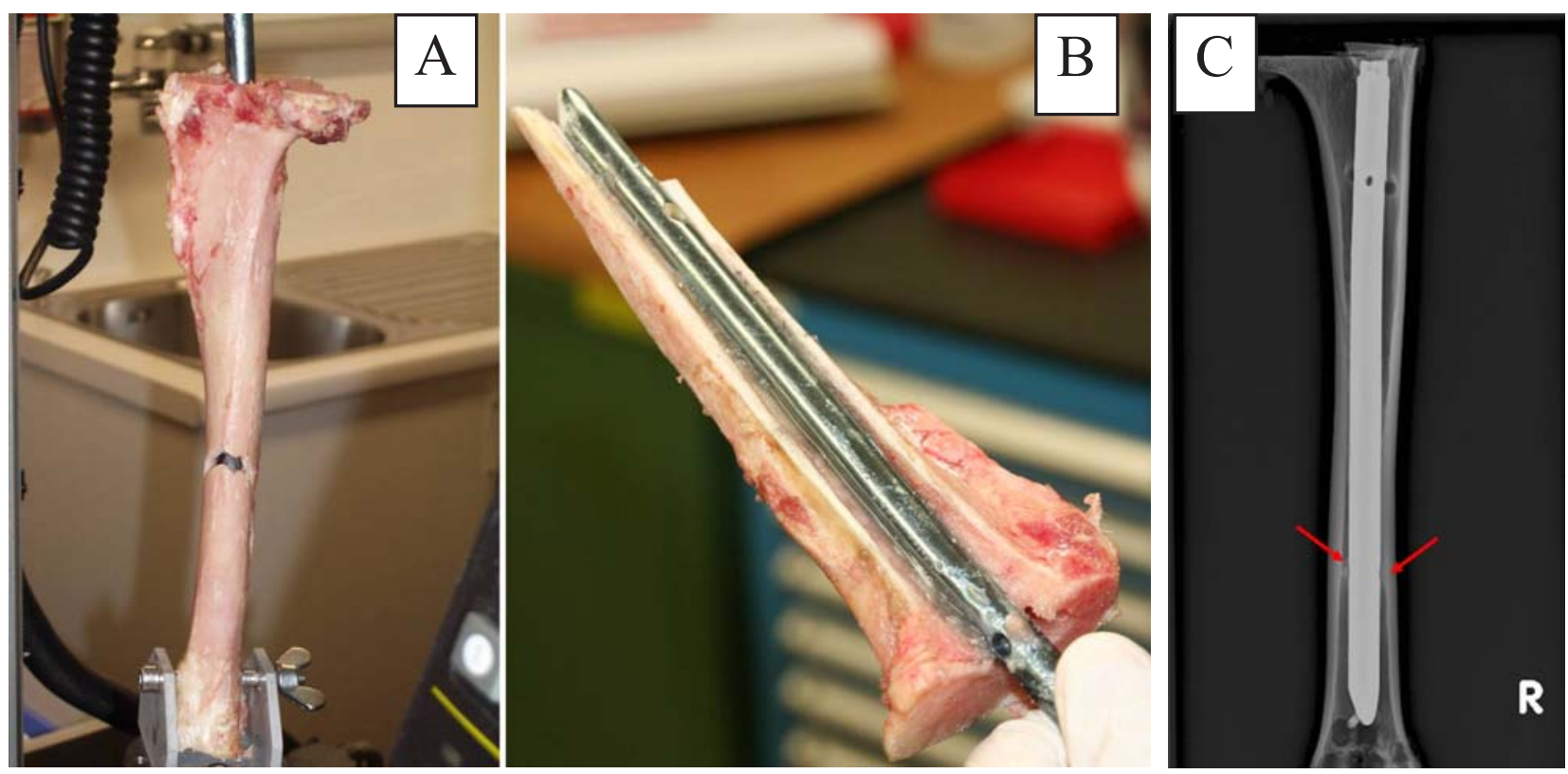

Figure 5. (A) One polished nail produced similar pull out strength to the standard TAN nail (denoted by purple dashed line in Fig. 4), as the bone broke during attempted extraction. (B) On later inspection we noted that the medullary canal containing the PP-TAN IM nail was extremely tight fitting despite reaming. The Universal Humeral Nails (UHN) used in this study is designed for humans therefore do not have an anatomically ideal shape for sheep IM canals. Despite a rigorous radiological analysis of the sheep IM canals prior to implantation, large sheep within the surgery flock were limited, therefore, it seems in this case, the IM canal was not suitable. (C) Bony in-growth into the empty distal screw hole was noted (red arrows), therefore the results for the pullout test was a reflection of the force to break a cylinder of the bone in the hole, rather than on the force required to remove the nail (denoted by blue dashed line in Figure 4). The data from this sheep was excluded from the statistical analysis.
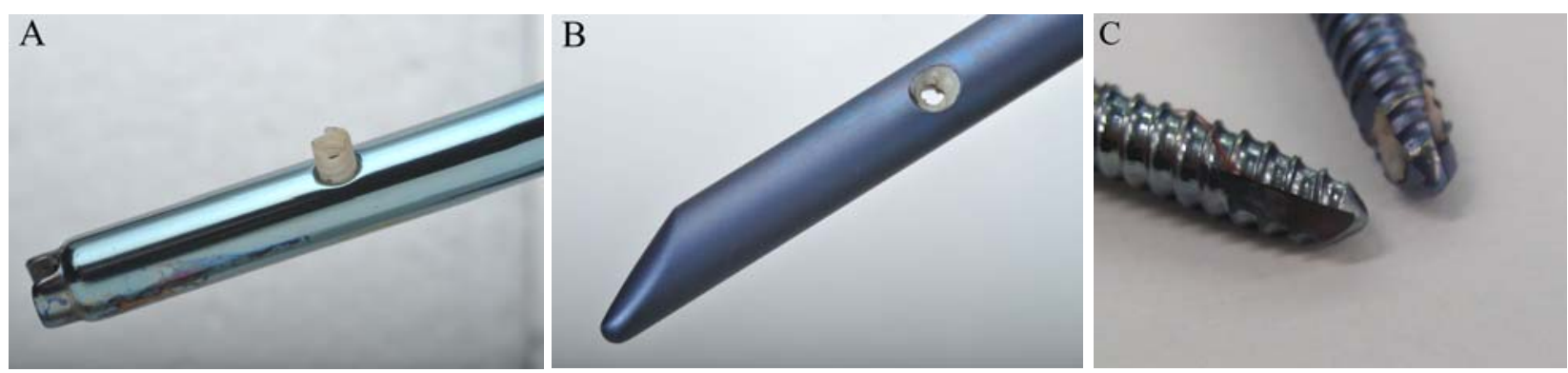

Figure 6. No bone adhesion on the nails was observed subsequent to removal. Within the distal interlocking holes of the polished nails (PP-TAN), bone in-growth was observed only with one nail, and any tissue within the proximal screw holes was easily displaced with a K wire (A). However, within the interlocking holes of standard TAN nails, bone in-growth was evident in three nails (B), and this hard tissue could not be displaced with a $\mathrm{K}$ wire (firmly bonded to the surface). Standard TAN proximal interlocking bolts also showed evidence of bone on-growth (C), while no tissue adhesion was observed for paste polished TAN interlocking bolts (light blue screw).

\section{Histological Observations}

In our proof of concept study that compared SS IM nails to TAN-S IM nails we observed direct bone bonding to the nail for TAN-S samples. Furthermore, direct bone contact to TAN-S was noted between the nail and the interlocking screw (Fig. 7A). In contrast, SS IM nails had a consistent fibrous layer around the circumference of the entire nail separating the nail from direct bone contact. This layer was observed to be only a few cell layers thick (Fig. 7B).

In our main study group aimed at testing the influence of surface polishing for ease of removal we noted that the direct bone bonding observed for TAN-S IM nails was not evident for TAN-PP IM nails. Similar to observations made in the previous group for SS IM nails, TAN-PP IM nails had a fibrous layer separating the implant from direct bone contact. However, it was noted that this fibrous layer was thicker than that noted for SS IM nails (Fig. 7C).

\section{Discussion}

To date, IM nails are fabricated for orthopaedic surgeons from either TAN or SS. Yet removal of standard TAN (STAN) IM nails often produces more extraction related complications compared SS IM nails of the same design 

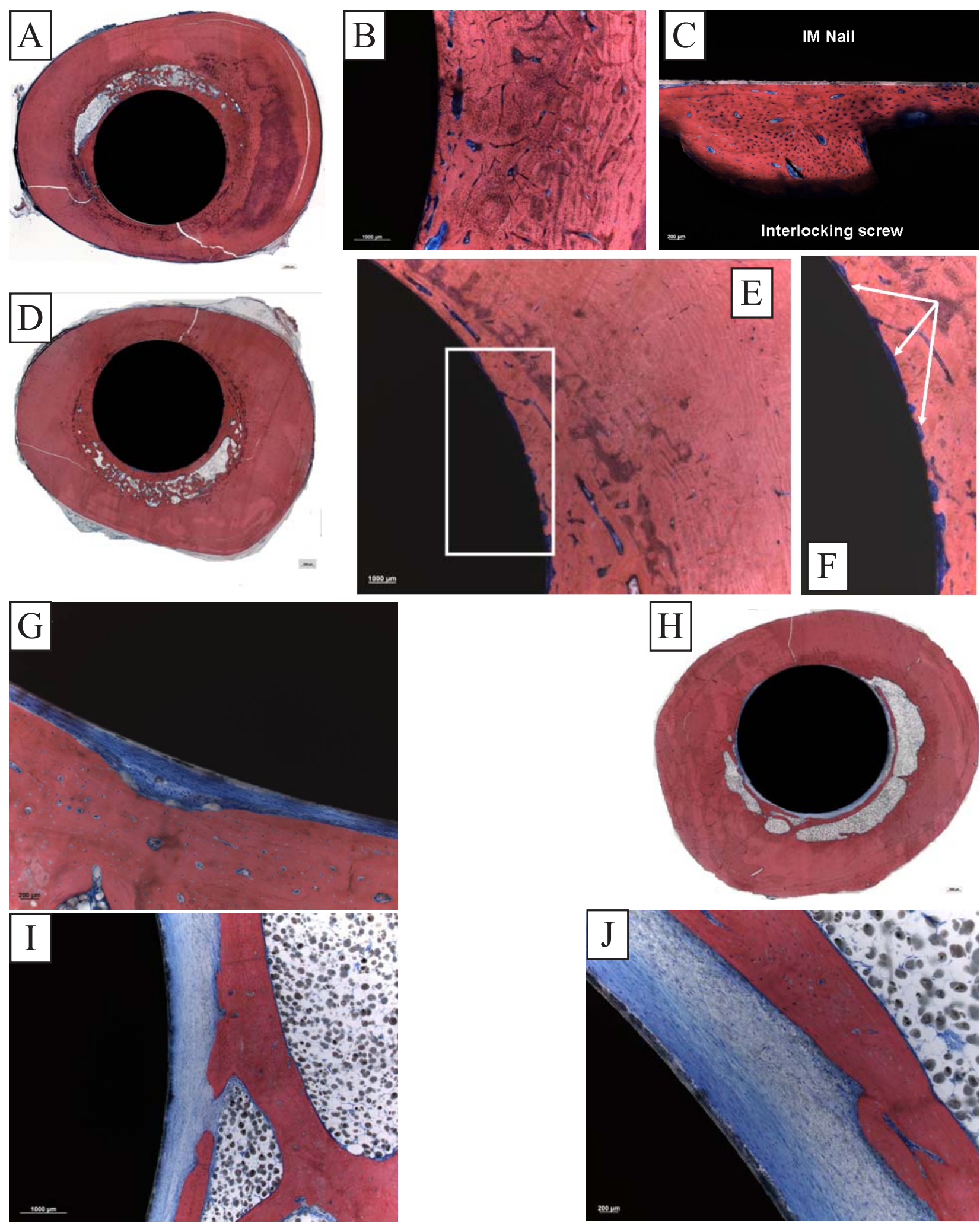

Figure 7. Representative histological sections of standard micro-rough TAN (A-C), stainless steel (D-G) and paste polished TAN (H-J) IM nails stained with Giemsa-Eosin to demonstrate soft tissue (blue) and bone (pink) attachment, after 12 months implantation. Note that with higher magnification, direct bone contact to the standard micro-rough TAN nail is evident adjacent the nail (B) and within the interlocking hole (C), despite the presence of the screw. It was evident for SS IM nails that a continuous fibrous layer (only a few cells thick) was present around the circumference of the nail (E). In higher magnification this layer clearly separates the implant from bone (white arrows) thereby preventing direct osseointegration (F (magnified area denoted by white box in $\mathbf{E}$ ) and $\mathbf{G}$ ). Similar to SS and in contrast to S-TAN, paste polished TAN IM nails appear to have a fibrous tissue layer separating the nail from the surrounding bone (I-J). No issues of stability were observed for SS or PP-TAN nails. This fibrous tissue layer is likely to contribute to the reduced pull-out strength required for these nails compared to standard micro-rough TAN nails 
(Im and Lee, 2003; Woodruff et al., 2003; Gösling et al., 2005). The difficulty in removing nails due to excess strong bone on-growth has not been described for stainless steel which for clinical orthopaedics is supplied with a smooth surface (Disegi, 1998a). In contrast, TAN has a microrough surface containing a micro-spiked surface morphology due to the fact that the alloy is a mix of soft alpha and harder beta phases (Sittig et al., 1999). Due to this surface morphology TAN has been shown to integrate extremely well with bone (Schmidt et al., 2002). Therefore, we believe that by reducing the surface microroughness that is associated with S-TAN IM nails by employing the mechanically abrasive technique of paste polishing that TAN IM nails will be easier to extract due to reduced capacity to support excessively strong bone on-growth. To do so we implanted SS and S-TAN IM nails in opposite sheep tibiae in one group and PP-TAN and STAN IM nails implanted within a paired non-fracture model in a separate group.

While the authors appreciate the value of reproducing the in vivo physiological milieu encountered in a fracture model the inclusion of a non-fracture model was chosen for several reasons. For instance, variations in healing such as non-union, mal-union and migration due to instability, were avoided. Additionally, the non-fracture model allowed for conclusions to be drawn specifically related to a material-dependent response rather than a biological response based on variations in healing. Also, since the medullary canal was extensively reamed essentially many of the same biological stimuli similar to those found in fracture healing were evoked. Importantly, however, within many countries ethical permission for a bilateral fracture would not be granted due to the implications to the animal. On top of this the increase in animal number which would be required to compensate for the loss of simultaneous comparisons within the same sheep and to allow for the statistical power of the study to be relevant would also not be ethically justified. A fracture model could only be applied unilaterally to prevent excessive pain and suffering to the animal therefore extrapolation of results from different sheep for the different materials would be incomparable due to inherent differences in healing and intramedullary canal size. Our study always compared the experimental version to the standard within the same sheep removing all inter-sheep variances such as IM canal diameter and other anatomical differences such as bone curvature, bone quality, etc.

The promise of surface polishing has been noted elsewhere, however, there have been few and inconsistent results to confirm this concept (Albrektsson and Hansson, 1986; Carlsson et al., 1988; Sun et al., 1999). Previous work in our laboratory has already highlighted the potential for surface polishing for controlling cellular response in vitro (Biggs et al., 2007; Meredith et al., 2007a; Meredith et al., 2007b; Hayes et al., 2008) as well extrapolating this to the in vivo situation (Richards et al., 2000; Welton, 2006; Pearce et al., 2008). Specifically in relation to hard tissue we have shown that standard micro-rough cpTi and TAN cortical screws had higher torque removal in both a trabecular and cortical sheep bone model compared to polished samples of the same materials over a period of 6 , 12 and 18 weeks (Pearce et al., 2008). However, despite the knowledge that surface microtopography can be a major determinant of bone osseointegration this avenue in terms of IM nailing technology has not previously been explored as a potential resolution to issues involving device removal. In fact, many studies suggest alternative methods for removal once conventional methods have failed (Alan et al., 2007; Weinrauch and Blakemore, 2007) such as alternative surgical approaches or use of surgical instruments in a different manner initially intended to aid removal; although this only addresses the issue once complications have been encountered. Instead, in this novel study we offer a simple and cost-effective method for reducing the problems associated with IM nail removal and resulting intra-operative complications due to excessive bony in-growth.

Results from this study support the indication that the surface microtopography of the IM nails has a significant effect upon the force required for removal. As excepted the pull out forces required for removal of SS IM nails was significantly lower than contra-lateral S-TAN IM nails implanted within the same sheep. Interestingly, by reducing the surface microtopography of the clinically available standard TAN IM nails we also observed a significant reduction in the force required for nail removal. As mentioned previously, TAN has a strong affinity to bone therefore, as the bulk material and oxide layer remain similar to that of S-TAN IM nails, as highlighted by chemical analysis of the surface using XPS, the fracture healing response should not be negatively affected. This has also been our experience with other polished devices fabricated from TAN, commercially pure titanium (cpTi) and titanium molybdenum (Richards et al., 2000; Welton, 2006; Pearce et al., 2008). Furthermore, radiologically no adverse influence of PP-TAN nails was observed throughout the implantation period of 12 months.

One may postulate that stainless steel already offers a solution without the need for further developments. However, issues surrounding toxic metal ion release (Case et al., 1994; Hanawa, 2004; Keegan et al., 2007) mechanical inferiority under cyclic loading (Eschbach, 2001; Perren et al., 2001), imaging disadvantages (Pohler, 2000; Eschbach, 2003) and second-rate biocompatibility (Massone et al., 1991; Nichols and Puleo, 1997) compared to TAN has resulted in its decreased potential within IM nailing technology.

Supporting this notion is the fact that there appears to be a material dependent outcome for extraction. Specifically, regardless of similar topographies stainless steel IM nails generally had lower extraction force needed for removal compared to paste polished TAN IM nails. This result may partially be attributable to the locking bolts which were included for all SS nails but not for PP-TAN and S-TAN nails. Essentially, this would minimise any tissue in-growth into the interlocking holes of the SS nails thus negating this effect on removal. However, the overriding contribution is likely to be a product of differences in the respective polished nails surface chemistry. Many studies have emerged that highlight the 
importance of the surface chemistry and in particular the importance of the oxide layer of fixation materials. Specifically, the surface chemistry of a device has been implicated in the initial protein-metal interactions (Meyer et al., 1988; Degasne et al., 1999; Yang et al., 2003) and it is believed that this relationship primes the material for subsequent cell phenotypic and genotypic performance and as a consequence ensuing tissue response. Essentially the bio-passivity of an implant is attributable to this oxide layer. Many alloying elements tend to show favourable properties in terms of low charged species at physiological $\mathrm{pH}$ with the likes of titanium, aluminium and niobium being accepted by tissue as 'inert' materials thus do not evoke adverse tissue reactions. However, other oxides have been reported to show either a preference to evoke a foreign body reaction resulting in a tendency to produce a fibrous capsule or indeed an outright toxic response (such as chromium and cobalt found in stainless steel). Iron oxide, another of the major elements found in SS, is known to induce encapsulation through its corrosion products and their solubility (Textor et al., 2001) while contrastingly cpTi and its alloys are known to have a superior corrosion resistance compared to SS (Disegi, 1998b). Due to the clear differences in oxide thickness between SS (approximately 1-2nm) and TAN (approximately 5nm) (Textor et al., 2001) the former affords itself more readily to corrosion than the latter especially since SS takes a longer period to repassivate thus inadvertently allowing increased time over TAN for metal ion release (Hanawa, 2004). The release of ions into surrounding tissue has previously been shown to play a role in periprosthetic pathology by contributing to implant failure by impairing bone repair while allowing fibrous tissue formation following debris-induced osteolysis (Nichols and Puleo, 1997). Therefore, one can speculate that the differences in surface oxide chemistry between SS and PP-TAN would produce different ultrastructural tissue responses that have been reported previously (Albrektsson and Hansson, 1986). Histological observations from this study challenge the overall assumption that direct bony integration is required for implant stability. Indeed considering the lack of published data describing direct osseointegration for stainless steel implants this concept does not come as any great surprise. In fact we and others have published data that distinctly show that stainless steel implants support a thin fibrous shell between bone and the device (Albrektsson and Hansson, 1986; Pearce et al., 2008; Hayes et al., 2009). Furthermore despite this distinct lack of direct osseointegration for steel vast quantities of stainless steel implants are sold annually without a significantly higher number of failure rates compared to titanium. Interestingly in this study we also observed indirect osseointegration of PP-TAN samples. Throughout the 12 month implantation period no problems of instability were noted again supporting the hypothesis that direct bony integration is not absolutely necessary for implant stability.

Finally, since infection remains one of the major limiting factors to successful fixation the effect of polishing on infection susceptibility is of paramount importance since essentially this is one factor that will decide if the application of this technology in clinics is feasible. To this end our group have previously shown that polishing TAN discs reduces in vitro bacterial adhesion in comparison with S-TAN (Harris et al., 2007). The effect of polishing on infection susceptibility is currently being tested in rabbits in vivo with these surfaces and materials.

\section{Conclusion}

Implant polishing holds great potential for IM nailing technology. Here we have shown that surface polishing reduces pull out forces required for nails which are significantly less compared to the clinically available standard TAN IM nails. Since TAN IM nails are generally preferred over SS nails due to their excellent biocompatibility and mechanical properties we believe these findings are extremely important for IM nails to help with insertion and removal.

\section{References}

Alan RK, Baig R, Voss FR (2007) Exchange femoral nailing: a new technique for removal of a broken nail. Am J Orthop 36: 500-502.

Albrektsson T, Hansson HA (1986) An ultrastructural characterization of the interface between bone and sputtered titanium or stainless steel surfaces. Biomaterials 7: 201-205.

Andrade JD (1985) The contact angle and interface energetics. Chapter 7. In: Surface and Interfacial Aspects of Biomedical Polymers: Surface Chemistry and Physics, vol. 1 (Andrade JD, ed). Plenum Press, New York and London.

Baxter LC, Frauchiger V, Textor M, ap Gwynn I, Richards RG (2002) Fibroblast and osteoblast adhesion and morphology on calcium phosphate surfaces. Eur Cell Mater 4: 1-17.

Biggs MJ, Richards RG, Gadegaard N, Wilkinson CD, Dalby MJ (2007) The effects of nanoscale pits on primary human osteoblast adhesion formation and cellular spreading. J Mater Sci Mater Med 18: 399-404.

Boerger TO, Patel G, Murphy JP (1999) Is routine removal of intramedullary nails justified? Injury 30: 7981.

Bombaci H, Gorgec M (2003) Difficulty in removal of intramedullary nails: The geometry of the distal end of the nail. Yonsei Med J 44: 1083-1086.

Boyan BD, Dean DD, Lohmann CH, Cochran DL, Sylvia VL, Schwartz Z (2001) The titanium-bone interface in vitro: The role of the surface in promoting osseointegration. In: Titanium in Medicine (Brunette DM, Tengvall $\mathrm{P}$, Textor M, Thomsen P, eds). Chapter 17, pp 561-586, Springer, New York.

Carlsson L, Rostlund T, Albrektsson B, Albrektsson T (1988) Removal torques for polished and rough titanium implants. Int J Oral Maxillofac Implants 3: 21-24.

Case CP, Langkamer VG, James C, Palmer MR, Kemp AJ, Heap PF, Solomon, L (1994) Widespread dissemination 
of metal debris from implants. J Bone Joint Surg Br 76: 701-712.

Degasne I, Basle MF, Demais V, Hure G, Lesourd M, Grolleau B, Mercier L, Chappard D (1999) Effects of roughness, fibronectin and vitronectin on attachment, spreading, and proliferation of human osteoblast-like cells (SaOs-2) on titanium surfaces. Calcif Tissue Int 64: 499507.

Disegi J (1988a) AO ASIF Wrought 18\% Chromium14\% Nickel-2.5\% Molybdenum Stainless steel implant material. AO ASIF Technical Commission Pamphlet, 1st ed.

Disegi, J (1988b) AO ASIF unalloyed titanium implant material. AO ASIF Technical Commission Pamphlet, 4th ed.

Eschbach, L (2003) 10 Frequently asked questions about magnetic resonance imaging in patients with metal implants. AO ASIF Materials Expert Group..

Eschbach L, Bigolin G, Hirsiger W, Gasser B (2001) Low-nickel steel for small bone fragment fixation plates and screws. Proc MAEG Meeting, Lausanne, Feb 2001.

Flynn JM, Luedtke LM, Ganley TJ, Dawson J, Davidson RS, Dormans JP, Ecker ML, Gregg JR, Horn BD, Drummond DS (2004) Comparison of titanium elastic nails with traction and a spica cast to treat femoral fractures in children. J Bone Joint Surg Am 86-A: 770-777.

Gogi N, Khan SA, Varshney MK (2006) Limb length discrepancy following titanium elastic nailing in paediatric femoral shaft fractures. Acta Orthop Belg 72: 154-158

Gösling T, Hufner T, Hankemeier S, Zelle BA, MullerHeine A, Krettek C (2004) Femoral nail removal should be restricted in asymptomatic patients. Clin Orthop Relat Res 423: 222-226.

Gösling T, Hüfner T, Hankemeier S, Richter M, Krettek C (2005) Indikation zur Entfernung von Tibiamarknägeln (Indication fort the removal of tibial marrow nails). Chirurg 76: 789-794.

Hanawa T (2004) Metal ion release from metal implants. Mat Sci Eng 24: 745-752.

Harris LG, Meredith DO, Eschbach L, Richards RG (2007) Staphylococcus aureus adhesion to standard microrough and electropolished implant materials. J Mater Sci Mater Med 18: 1151-1156.

Hayes JS, Archer, CW, Richards RG (2007) Reducing bone encasement of metal implants for elective removal. Trans 53rd ORS, San Diego, p 1630.

Hayes JS, Archer CW, Richards RG (2008) An in vitro evaluation for understanding the role of surface microtopography in controlling tissue integration. Proc World Biomaterials Congress, Amsterdam.

Hayes JS, Seidenglanz U, Pearce SG, Archer CW, Richards RG (2009) Reducing implant removal related morbidity with surface polishing - a novel in vivo study. Proc Orthopaedic Research Society, Las Vegas.

Hui C, Jorgensen I, Buckley R, Fick G (2007) Incidence of intramedullary nail removal after femoral shaft fracture healing. Can J Surg 50: 13-18.

Husain A, Pollak AN, Moehring HD, Olson SA, Chapman MW (1996) Removal of intramedullary nails from the femur: a review of 45 cases. J Orthop Trauma 10: $560-562$.
Im GI, Lee KB (2003) Difficulties in removing ACE tibial intramedullary nail. Int Orthop 27: 355-358

Karladani AH, Ericsson PA, Granhed H, Karlsson L, Nyberg P (2007) Tibial intramedullary nails - should they be removed? A retrospective study of 71 patients. Acta Orthop 78: 668-671.

Keegan GM, Learmonth ID, Case CP (2007) Orthopaedic metals and their potential toxicity in the arthroplasty patient: A review of current knowledge and future strategies. J Bone Joint Surg Br 89: 567-73

Luhmann SJ, Schootman M, Schoenecker PL, Dobbs MB, Gordon JE (2003) Complications of titanium elastic nails for pediatric femoral shaft fractures. J Pediatr Orthop 23: 443-447.

Massone L, Anonide A, Borghi S, Isola V (1991) Positive patch test reactions to nickel, cobalt, and potassium dichromate in a series of 576 patients. Cutis 47: 119-122.

Meredith DO, Eschbach L, Riehle MO, Curtis AS, Richards RG (2007a) Microtopography of metal surfaces influence fibroblast growth by modifying cell shape, cytoskeleton, and adhesion. J Orthop Res 25: 1523-1533.

Meredith DO, Riehle MO, Curtis AS, Richards RG (2007b) Is surface chemical composition important for orthopaedic implant materials? J Mater Sci Mater Med 18: $405-413$.

Meyer AE, Baier RE, Natiella JR, Meenaghan MA (1988) Investigation of tissue/implant interactions during the first two hours of implantation. J Oral Implantol 14: 363-379.

Miettinen H, Mäkelä A, Rokkanen P, Törmälä P, Vainio J (1992) Fixation of diaphyseal femoral osteotomy with self-reinforced biodegradable intramedullary implants: an experimental study on growing dogs. Clin Mater 9: 3136.

Morshed S, Humphrey M, Corrales LA, Millett M, Hoffinger SA (2007) Retention of flexible intramedullary nails following treatment of pediatric femur fractures. Arch Orthop Trauma Surg 127: 509-514.

Mutimer J, Hammett RD, Eldridge JD (2007) Assessing leg length discrepancy following elastic stable intramedullary nailing for paediatric femoral diaphyseal fractures. Arch Orthop Trauma Surg 127: 325-330.

Nichols KG, Puleo DA (1997) Effect of metal ions on the formation and function of osteoclastic cells in vitro. J. Biomed. Mater Res 35: 265-271.

Pearce AI, Pearce SG, Schwieger K, Milz S, Schneider E, Archer CW, Richards RG (2008) Effect of surface topography on removal of cortical bone screws in a novel sheep model. J Orthop Res 26: 1377-1383.

Perren SM, Pohler O, Schneider E (2001) Titanium as an implant material for osteosynthesis applications. In: Titanium in Medicine (Brunette DM, Tengvall P, Textor M, Thomsen P, eds), Chapter 23, pp771-823, Springer, New York.

Peterson HA (2005) Metallic implant removal in children. J Paediatr Orthop 25: 107-115.

Pohler OEM (2000) Unalloyed titanium for implants in bone surgery. Injury 31: 7-13.

Richards RG, Persson A, Gasser B, Wieling R (2000) Influence of surface microtopography on formation of 
capsules: An in vivo study of stainless steel implants in rabbits. $10^{\text {th }}$ Annual Conference European Orhtopaedic Research Society, Wiesbaden, October 2000; Transactions 10: 0-136.

Roure P, Ip WY, Lu W, Chow SP, Gogolewski S (1999) Intramedullary fixation by resorbable rods in a comminuted phalangeal fracture model. A biomechanical study. J Hand Surg [Br] 24: 476-481.

Schmidt C, Kaspar D, Sarkar MR, Claes LE, Ignatius AA(2002) A scanning electron microscopy study of human osteoblast morphology on five orthopedic metals. J Biomed Mater Res 63: 252-261.

Seligson D, Howard PA, Martin R (1997) Difficulty in removal of certain intramedullary nails. Clin Orthop Relat Res 340: 202-206.

Schlegel P, Hayes JS, Frauchiger VM, Wieling R, Textor M, Richards RG (2009) An in vivo evaluation of the biocompatibility of anodic plasma chemical treatment with calcium phosphate of titanium. J Biomed Mater Res B Appl Biomater 90: 26-34

Schneider GB, Perinpanayagam H, Clegg M, Zaharias R, Seabold D, Keller J, Stanford C (2003) Implant surface roughness affects osteoblast gene expression. J Dent Res 82: 372-376.

Simanovsky N, Tair MA, Simanovsky N, Porat S (2006) Removal of flexible titanium nails in children. J Pediatr Orthop 26: 188-192.

Sittig C, Hahner G, Marti A, Textor M, Spencer ND, Hauert R (1999) The implant material, Ti6Al7Nb: surface microstructure, composition and properties. J Mater Sci Mater Med 10: 191-198.

Sun C, Huang G, Christensen FB, Dalstra M, Overgaard S, Bunger C (1999) Mechanical and histological analysis of bone-pedicle screw interface in vivo: titanium versus stainless steel. Chin Med J (Engl ) 112: 456-460.

Textor M, Sittig C, Frauchiger V, Tosatti S, Brunette DM (2001) Properties and biological significance of natural oxide films on titanium and its alloys In: Titanium in Medicine (Brunette DM, Tengvall P, Textor M, Thomsen P, eds), Chapter 7, pp 171-230, Springer, New York.

Toms AD, Morgan-Jones RL, Spencer-Jones R (1996) Intramedullary femoral nailing: removing the nail improves subjective outcome. J Orthop Trauma 10: 560-562.

Vierhout BP, Sleeboom C, Aronson DC, Van Walsum AD, Zijp G, Heij HA (2006) Long-term outcome of elastic stable intramedullary fixation (ESIF) of femoral fractures in children. Eur J Pediatr Surg 16: 432-437.

Woodruff MJ, Hanson JR, Shaw DL (2003) Intramedullary tibial nails: a novel approach to removal when the standard method is not possible. Injury 34: 789790.

Weinrauch PC, Blakemore M (2007) Extraction of intramedullary nails by proximal stacked wire technique. J Orthop Trauma 21: 663-664.

Welton JL (2006). In vivo evaluation of defined polished surfaces to prevent soft tissue adhesion. Master's Thesis, University of Wales, Aberystwyth.

Yang Y, Cavin R, Ong JL (2003) Protein adsorption on titanium surfaces and their effect on osteoblast attachment. J Biomed Mater Res A 67: 344-349.
Zenios M, Malik MHA, Al-Mesri, AR, Vhadra R, Khan SA (2004) Current intramedullary nail insertion and removal practice in the UK. Eur J Orthop Surg Traumatol 14: 19-22.

\section{Discussion with Reviewers}

Reviewer II: To what extent did the polishing process alter the surface roughness? Was this at a cellular level or larger dimensions?

Authors: Characterisation of the standard TAN IM nails indicate that surface roughness is approximately $1 \mu \mathrm{m}$. By applying paste polishing this roughness average was reduced to approximately $0.2 \mu \mathrm{m}$. This technique was very successful at reproducibly producing a smooth surface that was void of microdiscontinuities - so much so that even the beta phase particles characteristic of TAN were difficult to distinguish (as highlighted in figure $2 \mathrm{a} \& \mathrm{~b}$ ). Given the spectrum of roughness involved, we would suggest that these changes act initially on a cellular level to influence the bone-implant interface. We have recently published (RG Richards, The Relevance of Implant Surfaces in Hand Fracture Fixation In: Osteosynthesis in the Hand: Current Concepts. FESSH Instructional Course 2008. Basel, Karger,, pp 20-30) our hypothesis identifying an 'effective roughness spectrum' within which cells and tissues will strongly adhere or integrate to the surface. Cells do not react to roughnesses that are larger than they are which depending upon the cell type may be from $5 \mu \mathrm{m}$ upwards or around $50 \mu \mathrm{m}$ upwards. Based upon personal experience of working with cell adhesion in vitro and in vivo and personal interpretation of other works within topographical surface modification we suggest that cells will interact with strong effect for fracture fixation implants from $200 \mathrm{~nm} /$ $0.2 \mu \mathrm{m}$ to $2000 \mathrm{~nm} / 2 \mu \mathrm{m}$. Below the lower limit of this roughness spectrum cells have been observed to react in vitro but these changes have not been observed in vivo. This is likely because the proteins attaching to the surface upon implantation completely mask such fine topographies and their effect on the cells is masked. Furthermore, in previous in vitro work from our laboratory, working with these implant materials with similar roughness averages, we have identified a surface dependent outcome on rat calvarial osteoblast mRNA. Briefly, we have shown that these changes in microtopography due to polishing were sufficient to reduce significantly Osteocalcin mRNA compared to standard microrough counterparts over a 21 day culture period indicating that polished samples support a slower rate of terminal differentiation compared to standard microrough samples (or alternatively that terminal differentiation is accelerated on standard microrough samples). A manuscript describing this data has also been submitted to this journal and has been accepted for publication pending revision.

Reviewer II: Why was a 12 month post-operative time point selected?

Authors: The majority of planned implant removal procedures tend to occur between 6-18 months. This allows 
for adequate fracture healing to occur in patients prior to device removal. In many cases complete fracture healing has not occurred after 6 months. Therefore, we chose a 12 month post-operative time point to make the study clinically relevant in terms of time allowed for extraosseous formation.

Reviewer II: It is clear that bone tissue integration is not specifically desirable in the use of IM nails and the use of electropolished SS IM nails is the preferred method of fracture fixation. So why try to use a more biologically interactive material and then alter the surface to make them perform as well as SS nails?

Authors: We would not necessarily agree that the use of electropolished SS IM nails is the preferred method of fracture fixation. SS IM nails were initially introduced for IM nailing systems however the demand for low stiffness and high fatigue strength to prevent implant failure was met by changing from SS to TAN. While SS in theory has the ideal surface microtopography several other issues exist that limit its use in fracture fixation which resulted in TAN becoming the preferred material choice for IM nailing systems. For instance, despite providing an excellent and reproducible smooth surface, the oxide layer/passive film of SS is much thinner than that of titanium and its alloys and differs dramatically chemically. As a result of these differences SS implants re-passivate their surfaces at a significantly slower rate compared to TAN which involves an instantaneous reaction. Due to the slower rate of repassivation of SS there is a higher risk of metal ion/ particle release into surrounding tissue. Furthermore, particles from cpTi and its alloys comprise mostly insoluble titanium oxides which are recognised to be biologically inert. Tissue discoloration due to titanium oxide particles is sometimes seen around pure titanium implants, but this seems to have no clinical consequences. In contrast, components of SS such as chromium, cobalt, iron and nickel have been implicated as major contributors in the negative biological response evoked by metal particulates and salts and have emerged as potential hazards to the vascular, immune, excretory, reproductive, integumentary and nervous systems.

Some major differences also exist between the magnetic properties of stainless steel and cpTi and its alloys with the lowest susceptibility being observed for cpTi, TAN and Ti15Mo. Stainless steel reportedly produces artefact susceptibility ten times higher than that of cpTi. As the human body contains mostly water, implants with as close a magnetic susceptibility to water as possible produce the least amount of artefacts. Water has a magnetic susceptibility of 12.9710-6 while TAN is approximately 217.10-6 compared to 2200.10-6 for SS. Thus SS implants produce more significant image distortion compared to cpTi and its alloys. 